\title{
Retroperitoneoscopic Pancreatic Necrosectomy: Taming the Relentless March of the Great Marauder
}

\author{
Yash Rohatgi ${ }^{1, \odot}$ Abhijit Joshi ${ }^{1}$ \\ ${ }^{1}$ General Surgery, Laparoscopic Surgery, Dr. L.H. Hiranandani
Hospital, Powai, Mumbai, Maharashtra, India
}

\begin{abstract}
Address for correspondence Yash Rohatgi, MBBS, General Surgery, Laparoscopic Surgery, Dr. L.H. Hiranandani Hospital, 3rd Floor, Powai, Mumbai, Maharashtra 400076, India (e-mail: yash.rohatgi@gmail.com).
\end{abstract}

\author{
Abstract \\ Keywords \\ - acute pancreatitis \\ - retroperitoneoscopic \\ necrosectomy \\ - CT scan \\ - necrotizing \\ pancreatitis \\ - minimally invasive \\ pancreatic \\ necrosectomy
}

We hereby report a case of a 35-year-old male who presented to us with infected pancreatic necrosis, 4 weeks after an episode of acute alcoholic pancreatitis. Imaging investigations showed a predominantly left-sided pancreatic and peripancreatic infected collection. This case was managed successfully in a single sitting by retroperitoneoscopic intervention.

\section{Introduction}

The supreme art of war is to subdue the enemy without fighting. If you know the enemy and know yourself, you need not fear the result of a hundred battles. Sometimes we need to lose the small battles to win the war. Opportunities multiply as they are seized.

These pearls of wisdom were propounded by the Chinese general, writer, and philosopher, Sun Tzu, in his masterpiece on military strategy, The Art of War.

In many ways, managing a clinically worsening case of infected pancreatic necrosis (IPN) is like going to war. The multiple small battles that sometimes are part of a war are a grim reminder of the many ups and downs a patient of IPN has to go through during the course of this deadly disease.

\section{Case Report}

Our patient, a 35-year-old man, was initially admitted to a local nursing home with an acute abdomen. He was diagnosed to have acute alcoholic pancreatitis and was discharged after 4 days of conservative management once his symptoms improved.

published online

November 29, 2020
DOI https://doi.org/

$10.1055 / \mathrm{s}-0040-1721153$

ISSN 2455-7420.
Almost 4 weeks after that, he presented to our emergency department with complaints of left upper quadrant nonradiating acute pain, which was $7 / 10$ on the visual analog pain score. This was accompanied with nonbilious vomiting and fever.

On initial physical evaluation the pulse rate was 120 beats per minute, blood pressure of 150/90 $\mathrm{mm}$ of $\mathrm{Hg}$, temperature of $101^{\circ} \mathrm{F}$, and respiratory rate of 18 breaths per minute with an oxygen saturation of $98 \%$ on room air. A per abdomen examination revealed tenderness and distension in the left upper quadrant.

Laboratory findings revealed an elevated WBC count $(20,000)$ with $85 \%$ neutrophilia. Serum amylase and lipase were $650 \mathrm{U} / \mathrm{L}$ and 15,000 U/L, respectively, CRP was 280, serum procalcitonin was $1.98 \mathrm{ng} / \mathrm{mL}$, aspartate aminotransferase was $150 \mathrm{U} / \mathrm{L}$, alanine aminotransferase was $65 \mathrm{U} / \mathrm{L}$, and gamma-glutamyl transpeptidase was $120 \mathrm{U} / \mathrm{L}$, suggestive of pancreatitis and alcoholic liver disease.

A contrast-enhanced computed tomography (CECT; -Fig. 1) of the abdomen was suggestive of necrosis involving the distal body and tail of the pancreas (around 30-35\%)

(C) 2020. Medical and Surgical Update Society.

This is an open access article published by Thieme under the terms of the Creative Commons Attribution-NonDerivative-NonCommercial-License, permitting copying and reproduction so long as the original work is given appropriate credit. Contents may not be used for commercial purposes, or adapted, remixed, transformed or built upon. (https://creativecommons.org/licenses/by-nc-nd/4.0/) Thieme Medical and Scientific Publishers Pvt. Ltd., A-12, 2nd Floor, Sector 2, Noida-201301 UP, India 

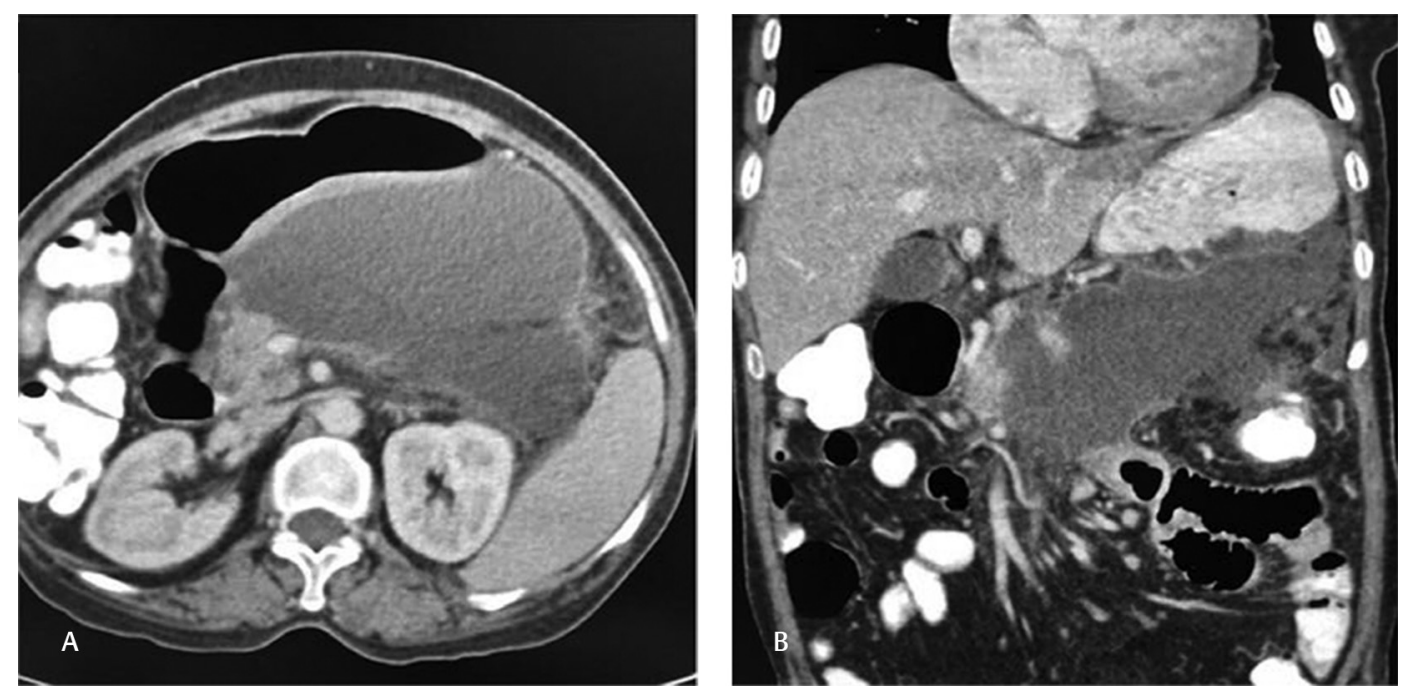

Fig. 1 Contrast-enhanced computed tomography of the abdomen. (A) Axial view and (B) coronal view depicting distal pancreatic necrosis with peripancreatic collection.

along with a large peripancreatic fluid collection, with small multiple gas pockets and necrotic debris within, and peripancreatic fat stranding, predominantly in the left half of the abdomen.

Thus, he was diagnosed to have IPN. He was admitted to the intensive care unit, kept nil by mouth with continuous nasogastric tube aspiration, started on intravenous fluids, antibiotics (cefoperazone $3 \mathrm{~g}$ intravenous [IV] loading dose followed by $1.5 \mathrm{~g}$ twice a day and metronidazole $500 \mathrm{mg}$ IV twice a day), and was planned for surgery.

He underwent retroperitoneoscopic necrosectomy with the aim to remove the necrotic debris, suck out the infected collection, give local toilet, and leave drainage tubes in situ for continuous postoperative toilet and drainage.

The patient was placed in the right lateral decubitus position, and the table was broken in the middle so as to raise the loin and improve surgical access. A $1.5-\mathrm{cm}$ incision was made just below the tip of the 12th rib on the posterior axillary line and was deepened in layers till the retroperitoneal space was reached. Then the index finger was inserted into this space to sweep the peritoneum anteriorly. Thereafter a $10-\mathrm{mm}$ optic trocar was inserted, and retroperitoneal working space was further developed using insufflation pressure and careful 0-degree telescopic dissection under camera vision. After this, a second $10-\mathrm{mm}$ trocar was inserted in the renal angle under direct vision. A third 5-mm trocar was inserted just above the iliac crest on the midaxillary line - Fig. 2. This ensured optimum triangulation. A suction irrigator and nontraumatic grasper were introduced through the working trocars. Pus and necrotic debris were then evacuated, -Figs. $\mathbf{3}$ and $\mathbf{4}$ a copious toilet was given with normal saline, and two 32-French tube drains were introduced through the $10-\mathrm{mm}$ trocar sites and fixed in situ. A sample of the sucked out pus was sent to the laboratory for culture study.

He had an uneventful postoperative course following the surgery and was discharged on postoperative day 7. Over a six monthly follow-up period, he was mostly symptom-free and was referred to our deaddiction clinic thereafter.

\section{Discussion}

Necrotizing pancreatitis (NP) entails necrosis or death of pancreatic parenchyma in varying degrees. The necrosed area is sterile to begin with but may get infected as the disease progresses. IPN occurs in approximately $30 \% 1$ of patients with NP. It is the most feared surgical complication of acute pancreatitis (AP). Once it develops, mortality rate increases significantly, as it can further lead to multiple organ dysfunction syndrome.

The Revised Atlanta Classification (RAC) ${ }^{2}$ categorizes AP into the following three types: (1) mild acute, (2) moderately severe acute, and (3) severe acute, depending on the presence or absence of local or systemic complications \& transient or persistent organ failure (OF). In addition, it classifies intraand peripancreatic collections into the following three types: (1) acute pancreatic fluid collections, (2) pseudocyst of the pancreas, (3) acute necrotic collections, and (4) walled-off pancreatic necrosis, depending on whether the collection is less or more than four weeks old and depending on the presence or absence of necrotic debris within it.

One of the shortcomings or criticisms of the RAC is that it has not given due importance to infected necrosis (the subject of this paper) while classifying the severity of AP. Tenner et al observed that though there was no difference in the development of OF with respect to IPN, the death rate was higher in patients with IPN. ${ }^{3}$ Isenmann et al, ${ }^{4}$ Büchler et $a l^{5}{ }^{5}$ and Le Mée et $\mathrm{al}^{6}$ noticed that OF was more frequent in patients with IPN than in those with sterile necrosis (SN) and that patients with IPN had higher mortality compared with those with SN.

The diagnostic indicators of NP and IPN are listed as follows. Clinical parameters are unreliable as they may anyway be deranged because of the systemic inflammatory response syndrome caused by acute non-NP as well as sterile NP:

- Culture study of a sample of the suspicious collection obtained by its image-guided aspiration grows the culprit bacterium. 


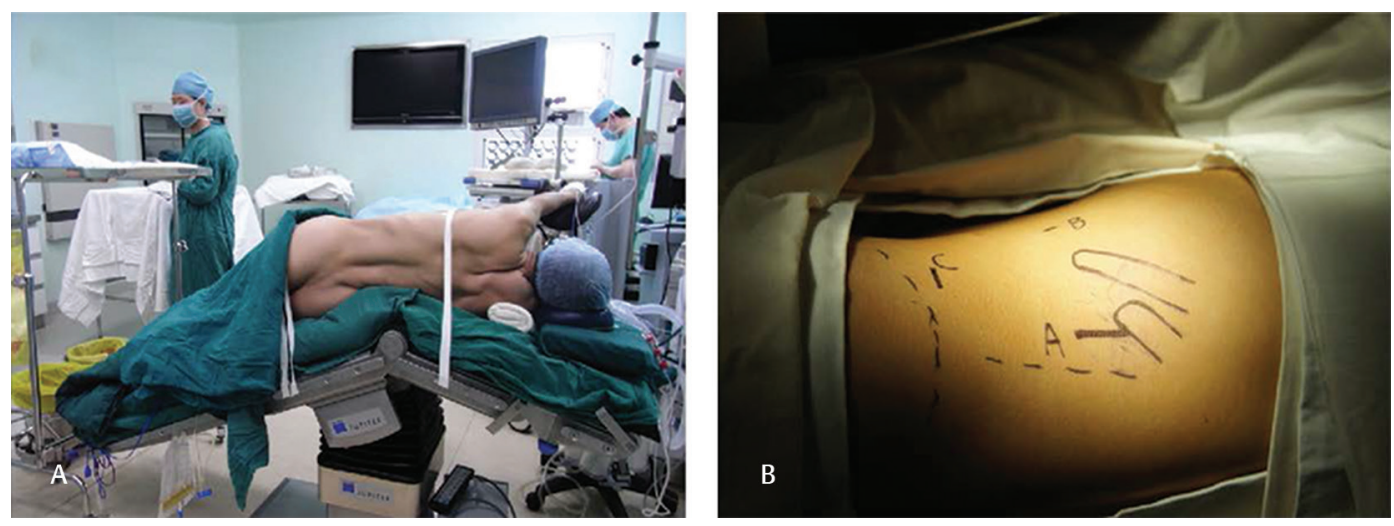

Fig. 2 (A) Patient position during retroperitoneoscopic pancreatic necrosectomy. (B) Trocar insertion sites depicted in the image.
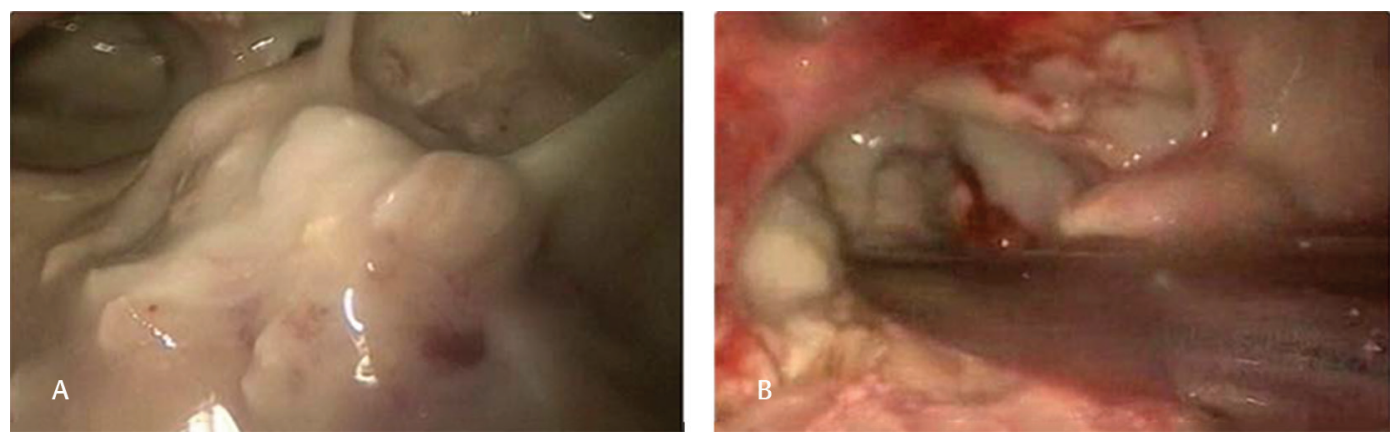

Fig. 3 (A) Liquified pus and thick pus flakes observed in the retroperitoneum. (B) Pus being sucked out and flakes being removed.
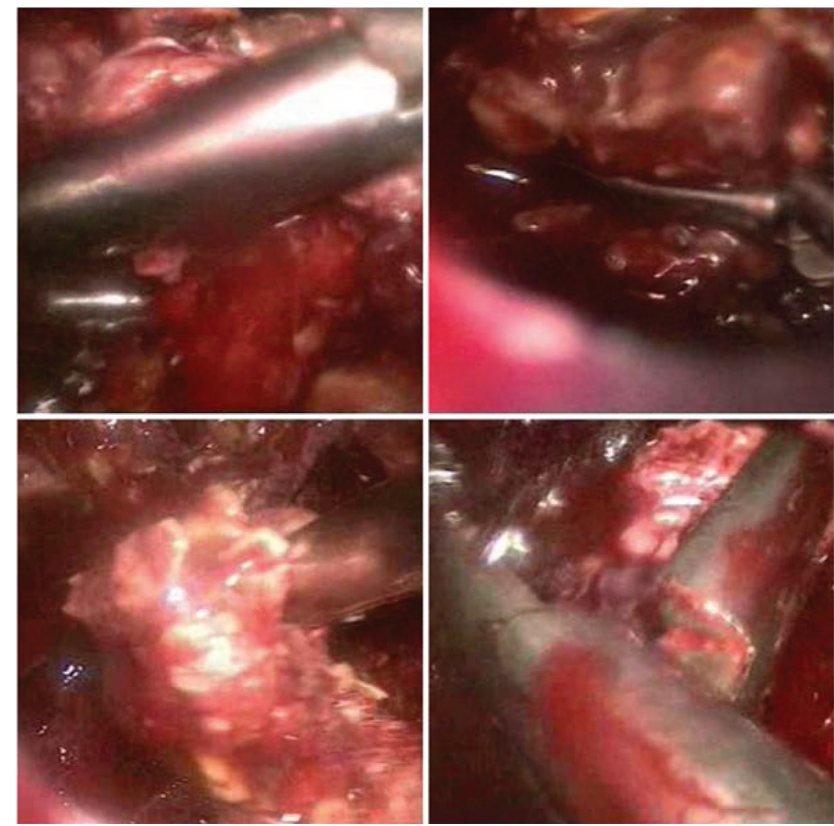

Fig. 4 Necrotic debris being removed.

- Grossly elevated serum procalcitonin levels.

- CECT shows air pockets in the collection.

- MRI of the abdomen and endosonography (EUS) are good modalities to diagnose and quantify necrotic debris within a collection. This helps in accurately classifying it and planning optimum treatment accordingly.
- Magnetic resonance cholangiopancreatography (MRCP): in cases with refractory or recurrent collections, a pancreatic ductal disruption is suspected. In such cases, MRCP (with prescan stimulation by secretin in some cases) is a good noninvasive diagnostic tool.

\section{Therapeutic Options in Infected Pancreatic Necrosis}

While outlining and enlisting the interventional options in the following, we would like to underscore the importance of clinical and investigational monitoring systems such as the Ranson's score, APACHE-2 (Acute Physiologic Assessment and Chronic Health Evaluation 2) score, Glasgow score, BISAP (bedside index of severity in acute pancreatitis) score, and CT severity index as the continual intensive watchdogs over this rapidly evolving, dynamic, and deadly disease.

- CT-guided percutaneous tube drainage: it has the advantage of being the least invasive and can be performed under local anesthesia (thus avoiding general anesthesia in a very sick patient). The material it provides is sent to the laboratory for culture study, and the antibiotics are tailored accordingly. The obvious disadvantage is that it is unable to drain necrotic debris, apart from the known problems of possible drain erosion into the bowel and formation of pancreaticocutaneaous fistula (PCF). ${ }^{7}$

- Endoscopic transmural necrosectomy: this has the advantage of avoiding drainage tube related bowel erosion and PCF. But it needs an expert in therapeutic endoscopy along with a well-equipped advanced therapeutic endoscopy 
setup. In addition, the prerequisite for it is that the infected collection abuts the posterior gastric or duodenal wall for the endoscopist to access it transmurally under EUS guidance. Thus, all other collections are not manageable with this option. ${ }^{8}$

- Transabdominal necrosectomy (transgastric, transmesocolic, or transgastrocolic). The advantage is minimal access and hence there are much lower chances of wound complications (infection, burst abdomen, etc.). Major disadvantages are limited operative field, complicated evacuation of necrotic tissue, contamination of peritoneal cavity, and, rarely, drain erosion into the bowel or formation of PCF.

- Retroperitoneoscopic necrosectomy: the major advantage of this approach over the transperitoneal one is avoidance of peritoneal contamination. The disadvantages are a learning curve and rare possibility of drain erosion into the bowel or formation of PCF. ${ }^{9}$

- Open necrosectomy: it has a high mortality (upto 25\%) and morbidity (upto 95\%) ${ }^{10}$ associated with it. It has been superseded by lesser invasive options enumerated previously in setups where these alternatives are available.

- Role of endoscopic retrograde cholangiopancreatography (ERCP) and stenting a pancreatic ductal disruption, if present, can be partial or complete. Patients with partial ductal disruption are greatly helped by ERCP and pancreatic ductal stenting. The stent bridges the area of disruption, seals it, and stops or minimizes the leak. Patients with complete ductal disruption (a very rare scenario) need surgery. ${ }^{7}$

\section{Controversies Related to Necrotizing Pancreatitis and Infected Pancreatic Necrosis}

Although IPN has been a well-known, well-studied, and well-researched entity for a long time, there still remain questions and controversies regarding its investigative modalities and management. These are as follows:

- Timing of CECT: if performed too early (within the first week), it may not show necrotic changes. Hence, it should ideally be performed after the first week so as to accurately diagnose NP early. ${ }^{11}$

- Timing of intervention for IPN: a conceptual change has taken place in this regard from an aggressive strategy in favor of early surgical intervention to a relatively conservative strategy in favor of delayed and minimally invasive intervention. Advocates of early surgery were in favor of the same to remove the focus of infection early so as to halt the inflammatory process. This was in keeping with time honored surgical teaching. However, it has been found that the human body's inflammatory responses to IPN are not only easily shut down but are also further augmented by early surgery. Surgeons all over the world have learned the hard way that early surgery is technically more challenging because the necrotic tissue is immature and is not easily separated from the viable tissue, thereby resulting in a significant risk of hemorrhage. Delayed surgery may permit time for stabilization of the patient and an easier removal of the well-demarcated necrosum. This being such a dynamic situation, the decision about optimum timing of intervention has to be individualized. A systematic review of published studies on the topic clarifies to us that the mortality is least when surgery is performed after 4 weeks. Nevertheless, the clinical picture (severity and progression) should always dictate the timing of intervention. ${ }^{12}$

- Selection of optimum sequence of interventional modalities: the need of the hour is evidence to guide selection of optimum sequence of interventional techniques for a particular patient and the timing of its execution. There is enough evidence in the literature to state that pancreatic necrosectomy by minimally invasive surgery is feasible and has a major role to play in reducing both systemic insult and the subsequent mortality. But it mandates technical expertise and a good technically sound setup. It may require multiple sessions as it is difficult to remove necrosum in a single sitting.

Trials on the subject are as follows:

- PANTER trial is a multicenter randomized controlled trial (RCT). It enrolled 88 patients and compared primary open necrosectomy versus step-up approach (percutaneous drainage followed by minimally invasive retroperitoneal necrosectomy). Mortality and morbidity were much lesser in the "step-up" group..$^{13}$

- POINTER trial is an ongoing multicenter RCT conducted by the Dutch pancreatitis study group. It has enrolled 104 patients and is comparing immediate catheter drainage (within the first 24 hours) versus postponed catheter drainage. Necrosectomy, if required, can be performed in both treatment arms.

- TENSION trial is a multicenter RCT that enrolled 98 patients and compared endoscopic intervention versus surgical step-up approach. It found similar morbidity/ mortality in both groups but lower incidence of fistulas in the endoscopy group. ${ }^{8}$

- PENGUIN trial was an RCT (22 patients) comparing endoscopic transgastric versus surgical necrosectomy. It concluded that the proinflammatory response (measured by S.IL6 levels) as well as composite clinical end point (major complications such as intra-abdominal bleeding, enterocutaneous or pancreatocutaneous fistula, or new-onset multiple OF) were lesser in the endoscopic group versus surgical group. ${ }^{13}$

\section{Conclusion}

Due to the aggressive nature of the disease, high rate of morbidity and mortality, variable outcomes of different modes of therapy in different patient populations, and different pancreatic medical and surgical units across the world, a standardized flowchart of management of this deadly disease still eludes us. The utopian goal of achieving the perfect balance between operating too early and too late is yet to be reached. The long wait continues as dedicated pancreatitis study groups around the world 
undertake more RCTs on the topic in an endeavor to answer the many questions left unanswered till present day.

Our patient did well in a single minimally invasive intervention most probably because he was young, had no comorbidities, and most likely did not have any major pancreatic ductal disruption.

\section{Conflict of Interest}

None declared.

\section{References}

1 Beger HG, Rau B, Mayer J, Pralle U. Natural course of acute pancreatitis. World J Surg 1997;21(2):130-135

2 Thoeni RF. The revised Atlanta classification of acute pancreatitis: its importance for the radiologist and its effect on treatment. Radiology 2012;262(3):751-764

3 TennerS, Sica G, Hughes M, et al. Relationship of necrosis to organ failure in severe acute pancreatitis. Gastroenterology 1997; 113(3):899-903

4 Isenmann R, Rau B, Beger HG. Bacterial infection and extent of necrosis are determinants of organ failure in patients with acute necrotizing pancreatitis. Br J Surg 1999;86(8):1020-1024

5 Büchler MW, Gloor B, Müller CA, Friess H, Seiler CA, Uhl W. Acute necrotizing pancreatitis: treatment strategy according to the status of infection. Ann Surg 2000;232(5):619-626

6 Le Mée J, Paye F, Sauvanet A, et al. Incidence and reversibility of organ failure in the course of sterile or infected necrotizing pancreatitis. Arch Surg 2001;136(12):1386-1390
7 Freeman ML, Werner J, van Santvoort HC, et al; International Multidisciplinary Panel of Speakers and Moderators. Interventions for necrotizing pancreatitis: summary of a multidisciplinary consensus conference. Pancreas 2012;41(8): 1176-1194

8 van Brunschot S, van Grinsven J, Voermans RP, et al; Dutch Pancreatitis Study Group. Transluminal endoscopic step-up approach versus minimally invasive surgical step-up approach in patients with infected necrotising pancreatitis (TENSION trial): design and rationale of a randomised controlled multicenter trial [ISRCTN09186711]. BMC Gastroenterol 2013; 13:161

9 Raraty MG, Halloran CM, Dodd S, et al. Minimal access retroperitoneal pancreatic necrosectomy: improvement in morbidity and mortality with a less invasive approach. Ann Surg 2010; 251(5):787-793

10 Werner J, Hartwig W, Hackert T, Büchler MW. Surgery in the treatment of acute pancreatitis-open pancreatic necrosectomy. Scand J Surg 2005;94(2):130-134

11 Balthazar EJ. Acute pancreatitis: assessment of severity with clinical and CT evaluation. Radiology 2002;223(3):603-613

12 Besselink MG, Verwer TJ, Schoenmaeckers EJ, et al. Timing of surgical intervention in necrotizing pancreatitis. Arch Surg 2007;142(12):1194-1201

13 van Santvoort HC, Besselink MG, Bakker OJ, et al; Dutch Pancreatitis Study Group. A step-up approach or open necrosectomy for necrotizing pancreatitis. N Engl J Med 2010; 362(16):1491-1502 